\title{
Cardiac resynchronization therapy in a patient with single ventricle and intracardiac conduction delay
}

\author{
Hideaki Senzaki, MD, Shunei Kyo, MD, Kazuo Matsumoto, MD, Haruhiko Asano, MD, Satoshi Masutani, MD, \\ Hirotaka Ishido, MD, Tamotu Matunaga, MD, Mio Taketatu, MD, Toshiki Kobayashi, MD, Nozomu Sasaki, MD, \\ and Yuji Yokote, MD, Saitama, Japan
}

ardiac resynchronization therapy (CRT) by left ventricular or biventricular pacing improves cardiac performance and clinical status in patients with heart failure and intraventricular conduction delay., ${ }^{1,2}$ This newer mode of heart failure therapy has previously only been used for patients with normal cardiac anatomy. Here we report the first case in which we applied this therapeutic methodology to a patient who had single-ventricle anatomy with advanced heart failure and intraventricular conduction delay.

\section{Clinical Summary}

The patient was an 18-year-old man with asplenia syndrome with cardiac anomaly associated with a single right ventricle, common atrioventricular (AV) valve, and pulmonary stenosis. Bidirectional Glenn anastomosis had been performed at a different institution when the patient was 8 years old, but this was subsequently reversed because of aneurysm formation at the anastomosis site. The patient had been followed up thereafter as having inoperable disease. He was repeatedly hospitalized because of worsening heart failure associated with severe AV valve regurgitation and cyanosis and was referred to our hospital. Initial examination revealed that the patient was in sinus rhythm and had right bundle branch block with a QRS duration of $160 \mathrm{~ms}$. Arterial oxygen saturation was $65 \%$, and ejection fraction was as low as $20 \%$. To evaluate whether surgery could improve the patient's clinical status and if so whether CRT could further improve ventricular performance in conjunction with the surgery, we performed cardiac catheterization (Figure 1, A). Right side (anterior wall) pacing alone raised maximum $\mathrm{dP} / \mathrm{dt}$ by $260 \mathrm{~mm} \mathrm{Hg} / \mathrm{s}$ and systolic blood pressure by $12 \mathrm{~mm} \mathrm{Hg}$. Adding left-side free wall (posterior wall) pacing further raised maximum $\mathrm{dP} / \mathrm{dt}$ by $61 \mathrm{~mm} \mathrm{Hg} / \mathrm{s}$ and systolic blood pressure by $7 \mathrm{~mm} \mathrm{Hg}$. However, left-side pacing alone had little effect on ventricular function. Pressure-area analysis ${ }^{3}$ revealed that both anterior wall and dual-site (anterior and posterior wall) pacing increased stroke work and lowered end-systolic vol-

From the Department of Pediatric Cardiology and Cardiovascular Surgery, Saitama Medical School Hospital, Saitama, Japan.

Received for publication July 1, 2003; accepted for publication Sept 16, 2003.

Address for reprints: Hideaki Senzaki, MD, Department of Pediatric Cardiology, Saitama Heart Institute, Saitama Medical School Hospital, 38 Morohongo, Moroyama, Saitama 350-0495, Japan (E-mail: hsenzaki@ saitama-med.ac.jp).

J Thorac Cardiovasc Surg 2004;127:287-8

$0022-5223 / \$ 30.00$

Copyright $\odot 2004$ by The American Association for Thoracic Surgery

doi:10.1016/j.jtcvs.2003.08.037 umes (Figure 2). Dual-site pacing enhanced end-systolic elastance from 5.7 to $10 \mathrm{~mm} \mathrm{Hg} / \mathrm{cm}^{2}$ and lowered effective arterial elastance from 25 to $18 \mathrm{~mm} \mathrm{Hg} / \mathrm{cm}^{2}$, indicating that pacing increased contractility and mechanical efficiency (Figure 2). AV delay had little influence on ventricular performance. Pulmonary artery pressure and resistance were $20 \mathrm{~mm} \mathrm{Hg}$ and $4 \mathrm{RU} / \mathrm{m}^{2}$, respectively. On the basis of these results, we placed epicardial pacing leads on the right and left sides of the single ventricle and on the atrial wall at the time of surgery for AV valve replacement and Blalock-Taussig shunt construction. The optimal pacing site was selected during surgery by determining which epicardial site provided the maximal rise in aortic pressure. The patient's condition markedly improved after the surgery, from New York Heart Association functional class IV to II. When VDD pacing was temporarily terminated for a few minutes, aortic pressure decreased by $20 \mathrm{~mm} \mathrm{Hg}$ with no change in heart rate, consistent with the results of cardiac catheterization. Simultaneously performed echocardiography revealed that the ejection fraction decreased from $45 \%$ to $24 \%$ with pacing cessation.

\section{Discussion}

To the best of our knowledge, this is the first report of successful CRT in a patient with single-ventricle anatomy and depressed ventricular contractility associated with intraventricular conduction delay. The mechanism by which left ventricular or biventricular pacing enhances cardiac function in patients with dilated cardiomyopathy is thought to be the synchronization of ventricular contraction. $^{2,4}$ By providing early stimulation to the otherwise late-activating region, pacing improves contractile coordination, thereby enhancing ejection. ${ }^{2,4}$ It is therefore reasonable that pacing an anatomic single ventricle with intraventricular conduction delay should enhance cardiac ejection by providing more coordinated contraction of the single ventricle. In patients with heart failure who have normal cardiac anatomy, left-side (left ventricular) pacing is required to maximize the efficacy of CRT. ${ }^{2}$ In patients with single ventricle, however, the optimal pacing site depends on the pattern of ventricular conduction and contraction. This patient had right bundle branch block, resulting in late activation of the right side (anterior) wall. Right-side pacing was therefore more effective than left-side pacing for our patient.

In addition to patients with an anatomic single ventricle, there are patients with congenital heart disease who have a functional single ventricle with two (right and left) anatomic ventricles, as is often observed after Fontan surgery. Interestingly, in such patients, asynchronous contraction between the two ventricles has been shown to induce hemodynamic deterioration. ${ }^{5}$ Synchronizing the contraction of such ventricles would enhance cardiac ejection by causing the ventricles to act as a single pump, and thus CRT may also be effective for improvement of cardiac performance in such cases. 

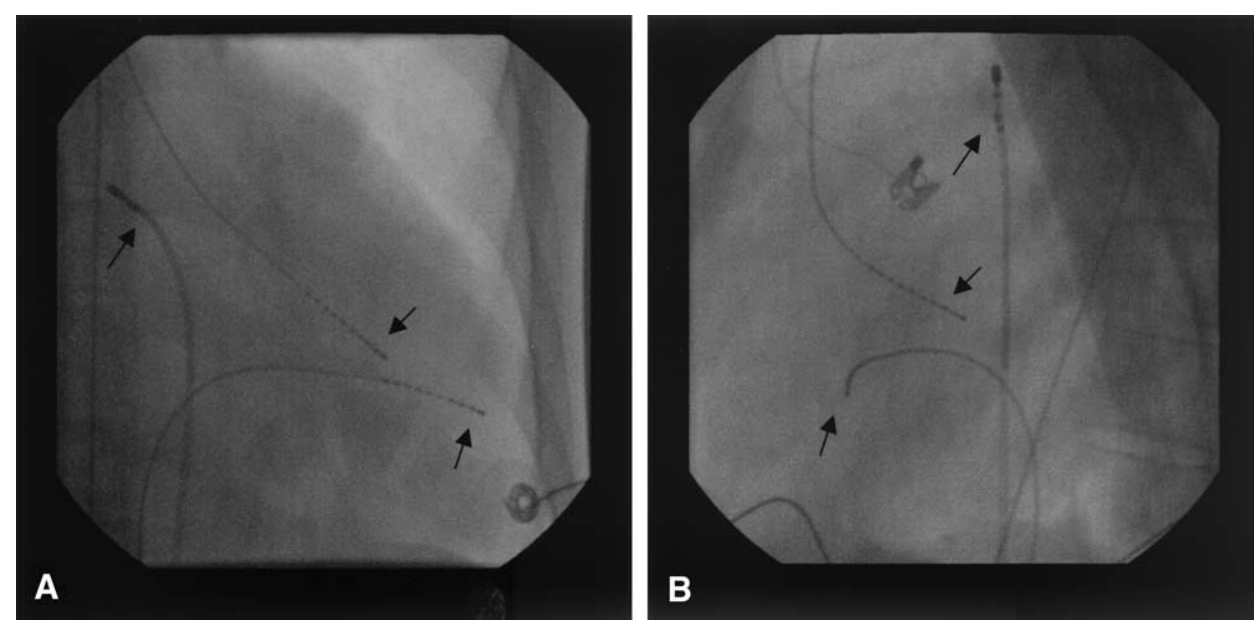

Figure 1. Anteroposterior (A) and lateral (B) views of ventricular pacing. Two pacing catheters in single ventricle and one atrial pacing catheter were inserted to test efficacy of CRT. Arrows indicate pacing catheters.

A

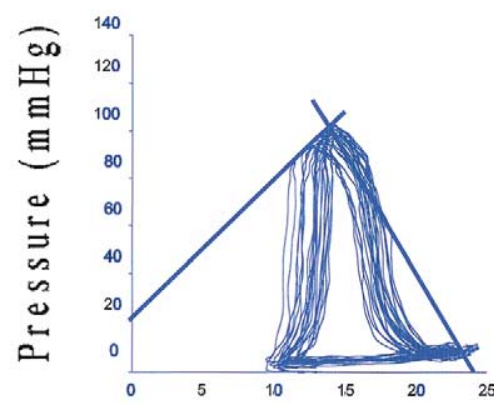

B

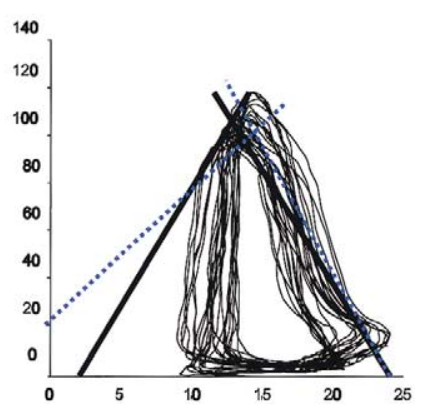

\section{Area $\left(\mathrm{c} \mathrm{m}^{2}\right)$}

Figure 2. Pressure-area loops and relationships before (A) and after (B) CRT by dual-site pacing. Dual-site pacing increased end-systolic elastance (solid lines) and lowered arterial elastance (solid lines). End-systolic and arterial elastance before pacing are reproduced (dashed lines) in part B to assist comparison.

In summary, there are increasing numbers of both pediatric and adult patients with heart failure associated with congenital cardiac anomalies, including single-ventricle anatomy. Our results suggest that CRT may be an effective treatment option for such patients with intraventricular conduction delay, independently or in conjunction with surgery for anatomic corrections.

\section{References}

1. Abraham WT, Fisher WG, Smith AL, Delurgio DB, Leon AR, Loh E, et al. Cardiac resynchronization in chronic heart failure. $N$ Engl J Med. 2002;346:1845-53.
2. Leclercq C, Kass DA. Retiming the failing heart: principles and current clinical status of cardiac resynchronization. J Am Coll Cardiol. 2002; 39:194-201

3. Senzaki H, Masutani S, Taketazu M, Kobayashi J, Kobayashi T, Sasaki $\mathrm{N}$, et al. Assessment of cardiovascular dynamics by pressure-area relations in pediatric patients with congenital heart disease. $J$ Thorac Cardiovasc Surg. 2001;22:535-47.

4. St John Sutton MG, Plappert T, Abraham WT, Smith AL, DeLurgio DB, Leon AR, et al. Effect of cardiac resynchronization therapy on left ventricular size and function in chronic heart failure. Circulation. 2003; 107:1985-90.

5. Yamamura H, Nakazawa M, Park I, Nakanishi T, Momma K, Imai Y. Asynchronous volume changes of the two ventricles after Fontan operation in patients with a biventricular heart. Heart Vessels. 1994;9:30714. 